\title{
Utilidad de la RMSSD-Slope para cuantificación de carga interna de entrenamiento en jugadores élite de bádminton. Estudio de caso Utility of the RMSSD-Slope for internal training load quantification in elite badminton players. Case study \\ *Marina Medina Corrales, *Alberto Garrido Esquivel, *Mariela Flores Cruz, *Flor Janeth Miranda Mendoza, *Myriam Zaraí García Dávila, *Germán Hernández Cruz, **José Naranjo Orellana *Universidad Autónoma de Nuevo León (México), **Universidad Pablo de Olavide (España)
}

Resumen: El objetivo de este estudio de caso fue verificar la utilidad de la pendiente de la raíz cuadrada de la media de las diferencias de la suma de los cuadrados entre intervalos RR adyacentes (RMSSD-Slope) como indicador individual de carga interna de entrenamiento en dos jugadores de Bádminton élite (un hombre y una mujer) durante 10 y 13 sesiones de entrenamiento respectivamente. Se realizaron registros de variabilidad de la frecuencia cardiaca durante cinco minutos antes y después de las sesiones de entrenamiento para calcular la RMSSD-Slope como indicador de carga interna, además de la escala de Borg. Se calculó el índice de estrés (SS) como indicador de actividad simpática, la RMSSD de actividad parasimpática y la relación entre ambos sistemas (Ratio S/PS). Los coeficientes de variación observados en los registros en reposo indican una alta variabilidad de las mediciones de cada día, lo que podría indicar las diferentes respuestas a las cargas de trabajo realizadas. No se encontró una relación lineal entre la RMSSD-Slope, RMSSD5, SS o variables de entrenamiento (volumen, intensidad y carga total de entrenamiento), sin embargo, se observó una correlación inversa entre la escala de Borg y la RMSSD-Slope. En conclusión, los resultados fundamentan el uso de la RMSSD-Slope y el SS como indicadores de la carga interna de entrenamiento que reflejan la respuesta individual de los atletas al estímulo. La utilización de la RMSSD-Slope podría ser un método práctico, no invasivo y de manejo sencillo que puede ser utilizado por los preparadores físicos y entrenadores.

Palabras clave:Variabilidad de la frecuencia Cardíaca, Carga de entrenamiento, RMSSD-Slope, Bádminton.

\begin{abstract}
The objective of this case study was to verify the utility of the slope of the mean of the differences in the sum of squares between adjacent RR intervals (RMSSD-Slope) as an individual indicator of internal training load in two elite badminton players (a man and a woman) during 10 and 13 training sessions respectively. Heart rate variability records were made for five minutes before and after training sessions to calculate RMSSD-Slope as internal load indicator, as well as the Borg scale. The Stress Score (SS) was calculated as an indicator of sympathetic activity, RMSSD of parasympathetic activity and the relationship between the two systems (Ratio S/PS). The coefficients of variation observed in the resting records indicate a high day-to-day measurement variability, which could indicate different responses to the workloads performed. Linear relationship was not found between the RMSSD-Slope and RMSSD5, SS or training variables (volume, intensity and total training load). Nevertheless, inverse correlation was observed between Borg scale and RMSSD-Slope. In conclusion, the case study results support the use of the RMSSD-Slope and the SS as indicators of the internal training load reflecting athletes' individual response to the stimulus. The use of the RMSSD-Slope could be a practical, non-invasive and easy-to-use method that can be used by physical trainers and trainers.
\end{abstract}

Keywords: Heart rate variability, Training load, RMSSD-Slope, Badminton.

\section{Introducción}

La carga de entrenamiento se puede cuantificar principalmente a través de dos indicadores, estos son la carga interna que se refiere a las reacciones individuales y específicas de cada atleta, provocadas por el entrenamiento y las cargas externas las cuales son representa-

Fecha recepción: 11-03-20. Fecha de aceptación: 13-10-20

Marina Medina Corrales

marina.medinacr@uanl.edu.mx das por las tareas de entrenamiento (Reina et al., 2019, 2020).

La variabilidad de la frecuencia cardiaca (VFC) permite realizar un análisis de manera no invasiva de la actividad del sistema nervioso autónomo (SNA)(Malik et al., 1996). En el ámbito del deporte, el análisis de la VFC es un instrumento que permite evaluar la adaptación a las cargas de entrenamiento deportivo por lo que facilita el seguimiento y control de los deportistas (Melanson \& Freedson, 2001; Tulppo et al., 2003).

Los métodos que se utilizan para la cuantificación de 
VFC pueden ser lineales y no lineales. Dentro de los métodos lineales, en el dominio del tiempo, se encuentra la RMSSD, que es la raíz cuadrada de la media de la suma de los cuadrados de las diferencias sucesivas de los intervalos RR. Esta medida es la más utilizada en el campo del deporte como indicador de la influencia parasimpática en el corazón (Buchheit, 2014). Se considera una medida de la variabilidad latido a latido y se puede utilizar como una medida de la variabilidad a corto plazo (Malik et al., 1996).

Por otro lado, se utilizan los métodos no lineales, entre los cuales la mayoría de los autores incluyen el gráfico de dispersión de Poincaré (Tulppo et al., 2003). Este gráfico se aproxima a una elipse y presenta un eje transversal (SD1), que refleja la actividad del sistema parasimpático y mide la variación de los intervalos $\mathrm{RR}$ a corto plazo, y un eje longitudinal (SD2) que refleja los cambios a largo plazo de los intervalos RR y se comporta de forma inversa a la actividad simpática (Tulppo et al., 1996).

El hecho de que SD2 resulte inverso a la actividad simpática supone algunos problemas en la interpretación de la relación SD1/SD2. Por ello, Naranjo et al., (2015) definieron dos nuevos conceptos a partir del gráfico de Poincaré: 1) el Stress Score o índice de estrés (SS), como inverso del SD2, cuya finalidad es obtener un valor proporcional a la actividad simpática sobre el nodo sinusal; 2) la ratio simpático-parasimpático (S/PS), como cociente entre SS y SD1, que refleja la relación entre la actividad simpática y parasimpática.

El deporte de Bádminton se caracteriza por acciones de alta intensidad de corta duración con gran componente del sistema anaeróbico (Cabello \& GonzálezBadillo, 2003), una frecuencia cardíaca máxima de 190,5 latidos por minuto y una media de 173,5 latidos por minuto durante partidos de más de 30 minutos de duración y la duración media de los puntos va de 6,4 segundos con tiempo de descanso de 12,9 segundos entre intercambios. Existen algunos estudios sobre el comportamiento de la VFC en jugadores de bádminton que nos indican la acumulación de fatiga durante los torneos (Garrido-Esquivel et al., 2009, 2011) y el posible papel predictivo sobre el éxito en competición (Bisschoff et al., 2018).

Garrido et al. (2009), estudiaron durante los XIV Campeonatos Panamericanos de bádminton (celebrados en México en 2007) a un jugador que llegó a la final, habiendo acumulado un total de cuatro partidos en 48 horas. Los autores describen que, tras cada partido, y a medida que incrementaba el número de encuentros, la
VFC se iba reduciendo. A partir de este caso, se analizó la VFC en reposo y después de tres partidos en 19 jugadores de bádminton, durante dos o tres días de competencia con el fin de valorar la fatiga acumulada. Para ello se analizó el comportamiento de los parámetros del dominio del tiempo y los diámetros SD1 y SD2 del gráfico de Poincaré. Los autores encontraron que los parámetros del dominio del tiempo medidos tras los partidos fueron significativamente más bajos que los de reposo (Garrido et al., 2011).

Por otra parte, un estudio realizado con un grupo de 22 jugadores de bádminton de élite refiere que las mediciones de VFC realizadas en el dominio de la frecuencia son predictores significativos para distinguir a los jugadores exitosos de los menos exitosos en un torneo (Bisschoff et al., 2018)

Sin embargo, no hay estudios en bádminton que analicen la carga interna de entrenamiento (CIE) en estos jugadores a partir de la VFC. Tradicionalmente la CIE se ha estimado con escalas subjetivas de percepción del esfuerzo de la sesión de entrenamiento (sRPE) (Foster, 1998) y por el impulso de entrenamiento (TRIMP) (Banister \& Calvert, 1980). La VFC, por su propio significado, podría ser una buena herramienta para valorar la carga interna pero no existían en la bibliografía propuestas útiles para valorar la recuperación tras ejercicio a partir de la VFC (McLaren et al., 2018). (Naranjo et al., 2019). En 2019 Naranjo et al., diseñaron un estudio en el que se propone el uso de la pendiente de recuperación de la RMSSD (RMSSD-slope) en los primeros 30 minutos posteriores a la actividad como indicador de carga interna. Para comprobar la utilidad de esta medida, posteriormente se realizaron mediciones retrospectivas de RMSSD-Slope en diferentes deportistas y en diferentes situaciones entre las que se encuentran algunos jugadores de bádminton (Ruso-Álvarez et al., 2019).

Por ello, el objetivo de este estudio de caso fue verificar la utilidad de la pendiente de la raíz cuadrada de la media de las diferencias de la suma de los cuadrados entre intervalos RR adyacentes (RMSSD-Slope) como indicador individual de carga interna de entrenamiento en dos jugadores de Bádminton élite (un hombre y una mujer) durante 10 y 13 sesiones de entrenamiento respectivamente.

\section{Material y método}

\section{Muestra}

Formaron parte de este estudio dos jugadores de bádminton de elite: un hombre (32 años, 78 kg, 182 
cm) y una mujer (22 años, $57 \mathrm{~kg}, 159 \mathrm{~cm}$ ), clasificados a juegos olímpicos de Tokio, se realizaron registros durante un total de 13 y 10 sesiones respectivamente.

Durante el periodo de estudio se solicitó a los sujetos abstenerse de consumir alcohol o productos con cafeína y antes de comenzar, se les proporcionó un cuestionario clínico para asegurar que no estaban recibiendo tratamientos farmacológicos o que no padecían ninguna enfermedad cardiovascular que pudiera alterar los registros. Los sujetos fueron previamente informados del procedimiento y dieron su consentimiento por escrito para participar en el estudio.

\section{Procedimiento}

Durante las sesiones de entrenamiento dentro del periodo competitivo, 10 en el caso de la mujer y 13 en el caso del hombre, se utilizó un monitor de FC Polar V800 (Polar Inc., Kempele, Finland) con un sensor torácico de FC Polar H7 (Polar Inc., Kempele, Finland). Se realizaron registros de VFC antes y después de las sesiones de entrenamiento, todos ellos de cinco minutos de duración. Los registros previos a la actividad no se hicieron en todas las sesiones, resultando válido un total de cuatro registros en el hombre y cinco en la mujer. Las mediciones posteriores al ejercicio se realizaron dentro de los primeros 30 minutos de finalizada la sesión de entrenamiento y descartando los primeros cinco minutos de recuperación por la pérdida de estabilidad en las series de tiempo debida al cambio entre el final del ejercicio y el inicio de la recuperación (Javorka et al., 2002).

Todas las mediciones se realizaron en posición de sentado. Para el registro posterior, se dio la instrucción a los sujetos de sentarse inmediatamente después de finalizar el entrenamiento y pasados cinco minutos se comenzaba con el registro.

Los intervalos RR se descargaron utilizando la aplicación Polar Flow Sync (Versión 3.0.0.1337, Kempele, Finland) para posteriormente ser analizados con el software Kubios HRV Standard (Versión 3.2.0, University of Eastern Finland, Kuopio, Finland). Cada registro fue previamente inspeccionado para detectar la posible presencia de irregularidades o artefactos, aplicando el filtro correspondiente en caso de ser necesario.

En cada sesión de entrenamiento se registró el volumen total, expresado en minutos, y la frecuencia cardiaca media de la sesión para calcular la intensidad media de la misma como porcentaje de la frecuencia cardiaca máxima (FCmáx). El producto de la intensidad por la duración se utilizó como valor de carga total de entrenamiento (Hayes \& Quinn, 2009).

Igualmente, en cada sesión de entrenamiento se recogió el valor correspondiente de la escala de Borg 1-10 (Borg, 1954) y se utilizó la RMSSD-Slope como indicador de carga interna de entrenamiento (Naranjo et al., 2019).

\section{Análisis de los datos}

En todos los registros (pre y post entrenamiento), tras el análisis de las series temporales en Kubios, se seleccionaron los datos correspondientes a RMSSD y gráfico de dispersión de Poincaré (SD1 y SD2). Con estos dos últimos datos se calculó el SS y la ratio S/PS, de acuerdo con el procedimiento propuesto por Naranjo et al. (2015). De esta forma pretendíamos disponer de un indicador de actividad simpática (SS), otro de actividad parasimpática (RMSSD) y la relación entre ambos sistemas (Ratio S/PS).

En el caso del registro posterior a la actividad se añadió el cálculo de la RMSSD-Slope. Para ello se utilizó el valor de RMSSD obtenido durante cinco minutos en los primeros 30 minutos de recuperación (RMSSD5) y se consideró un valor de RMSSD al final del ejercicio de cuatro milisegundos (Goldberger et al., 2006; Naranjo et al., 2019). La pendiente se calculó como (RMSSD5 - 4) / t; siendo t el tiempo de recuperación (en minutos) transcurrido desde el final de la sesión hasta el inicio del registro. Los valores obtenidos para la RMSSD-Slope se interpretaron de acuerdo a la propuesta realizada en relación a la intensidad (Naranjo et al., 2019).

Se presentan los valores de todas las variables para cada sujeto, facilitando la media, desviación estándar (DE) y el coeficiente de variación (CV) de cada medida.

Se realizaron análisis gráficos para determinar el grado de correlación entre RMSSD-Slope y las variables de volumen, intensidad y carga total de entrenamiento (CTE). Lo mismo se realizó con la RMSSD y el SS y entre el SS y la RMSSD-Slope.

Para estos cálculos se utilizó una hoja de Excel de Microsoft Office 365 ProPlus.

\section{Resultados}

Las Tablas 1 y 2 muestran los datos de los registros pre-entrenamiento de ambos sujetos, especificando la fecha y hora de registro, los valores de RMSSD y gráfico de dispersión de Poincaré (SD1 y SD2) obtenidos tras el análisis de las series temporales en Kubios, el 
cálculo de SS y la ratio S/PS, así como la media, DE y CV de todos los registros para cada variable.

Las Tablas 3 y 4 muestran los registros post-entrenamiento de ambos sujetos, en la que se expresan la FC media, el volumen total de la sesión expresado en minutos, la intensidad expresada en porcentaje, el valor de la carga total de entrenamiento, la escala de Borg 110, la RMSSD5, el SS, la ratio S/PS, la RMSSD-Slope y la interpretación de la misma.

Tabla 1

\begin{tabular}{|c|c|c|c|c|c|c|}
\hline \multicolumn{7}{|c|}{ RMSSD $(\mathrm{ms})$} \\
\hline 2 & $10: 15$ & 48.15 & 34.29 & 61.70 & 16.21 & 0.47 \\
\hline 5 & $10: 45$ & 69.34 & 49.43 & 104.60 & 9.56 & 0.19 \\
\hline 6 & $10: 44$ & 40.65 & 29.22 & 93.80 & 10.66 & 0.36 \\
\hline 7 & $10: 46$ & 42.72 & 30.67 & 118.47 & 8.44 & 0.28 \\
\hline \multirow[t]{3}{*}{8} & $10: 44$ & 33.33 & 24.06 & 121.52 & 8.23 & 0.34 \\
\hline & Media & 46.84 & 33.53 & 100.02 & 10.62 & 0.33 \\
\hline & DE & 13.65 & 9.62 & 24.14 & 3.27 & 0.10 \\
\hline \multicolumn{2}{|c|}{ Coeficiente de variación } & $29 \%$ & $29 \%$ & $24 \%$ & $31 \%$ & $32 \%$ \\
\hline
\end{tabular}

\begin{tabular}{cccccc} 
Coeficiente de variación & $29 \%$ & $29 \%$ & $24 \%$ & $31 \%$ & $32 \%$ \\
\hline RMSSD: Raíz cuadrada del valor medio de la suma de las diferencias al cuadrado de todos los
\end{tabular} intervalos RR; ms: milisegundos; SD1 y SD2: diámetro transversal y longitudinal respectivamente correspondientes al gráfico de Poincaré; SS: índice de estrés; ua: unidades arbitrarias; Ratio S/PS relación entre actividad simpática y parasimpática; DE: desviación estándar.

Tabla 2

\begin{tabular}{|c|c|c|c|c|c|c|}
\hline Sesión & Hora registro & $\operatorname{MSSD}(\mathrm{ms})$ & SD1(ms.) & SD2(ms.) & SS (ua) & Ratio $S / \mathrm{I}$ \\
\hline 2 & $10: 22$ & 52.92 & 37.68 & 63.51 & 15.75 & 0.42 \\
\hline 5 & $10: 51$ & 54.28 & 38.69 & 75.92 & 13.17 & 0.34 \\
\hline 7 & 10:39 & 56.18 & 40.04 & 90.26 & 11.08 & 0.28 \\
\hline 10 & $10: 38$ & 79.81 & 56.79 & 77.95 & 12.83 & 0.23 \\
\hline & Media & 60.80 & 43.30 & 76.91 & 13.21 & 0.32 \\
\hline & DE & 12.75 & 9.05 & 10.95 & 1.92 & 0.08 \\
\hline Coeficien & de variación & $21 \%$ & $21 \%$ & $14 \%$ & $15 \%$ & $26 \%$ \\
\hline
\end{tabular}
intervalos RR:ms. - milisegundos; SD1 y SD: diámetro transversal y longitudinal respectivamente correspondientes al gráfico de Poincaré; SS: índice de estrés; ua: unidades arbitrarias; Ratio S/PS relación entre actividad simpática y parasimpática; DE: desviación estándar.

Tabla 3

Regstro post-entrenamiento. Mujer

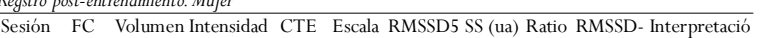

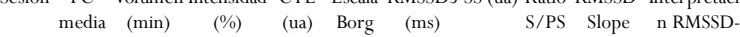

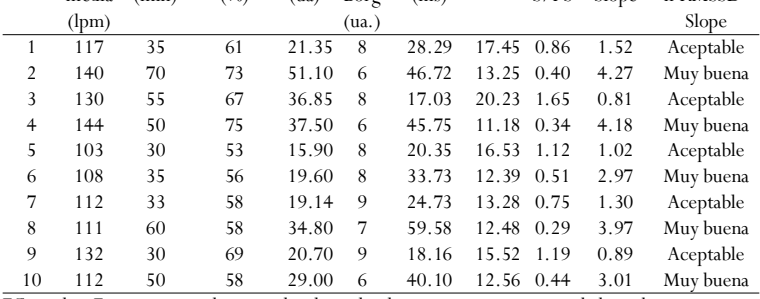
FC media: Frecuencia cardiaca media; lpm: latidos por minuto; ua: unidades arbitrarias; carg total de entrenamiento (CTE); Escala de Borg: escala de percepción del esfuerzo 1-10; RMSSD5: raíz cuadrada de la media de la suma de los cuadrados de las diferencias sucesivas de los intervalos RR en ms, registrada en un periodo de 5 minutos; SS: índice de estrés; Ratio S/PS: relación entre actividad simpática y parasimpática; RMSSD-Slope: pendiente de recuperación de la RMSSD.

Tabla 4

$\frac{\text { Registro post-entrenamiento. Hombre. }}{\text { FC }}$

\begin{tabular}{|c|c|c|c|c|c|c|c|c|c|c|}
\hline Sesión & $\begin{array}{c}\text { FC } \\
\text { media } \\
(\mathrm{lpm}) \\
\end{array}$ & $\begin{array}{c}\text { Volumen } \\
(\mathrm{min})\end{array}$ & $\begin{array}{l}\text { ntensidad } \\
(\%)\end{array}$ & $\begin{array}{l}\text { CTE } \\
\text { (ua) }\end{array}$ & $\begin{array}{c}\text { Escala } \\
\text { Borg } \\
\text { (ua) } \\
\end{array}$ & $\begin{array}{c}\text { RMSSD5 } \\
(\mathrm{ms})\end{array}$ & SS (ua) & $\begin{array}{l}\text { Ratio } \\
\text { S/PS }\end{array}$ & $\begin{array}{c}\text { RMSSD- } \\
\text { Slope }\end{array}$ & $\begin{array}{c}\text { Interpretació } \\
\text { n RMSSD- } \\
\text { Slope }\end{array}$ \\
\hline 1 & 132 & 35 & 71 & 24.85 & 8 & 9.19 & 54.02 & 8.22 & 0.52 & Aceptable \\
\hline 2 & 139 & 70 & 75 & 52.50 & 7 & 33.14 & 16.27 & 0.69 & 1.94 & Muy buena \\
\hline 3 & & 55 & 80 & 44.00 & 8 & 20.86 & 18.27 & 1.23 & 0.77 & Aceptable \\
\hline 4 & 148 & 50 & 80 & 40.00 & 8 & 36.39 & 15.30 & 0.59 & 2.02 & Muy buena \\
\hline 5 & & 30 & 80 & 24.00 & 8 & 8.55 & 27.59 & 4.49 & 0.46 & Aceptable \\
\hline 6 & 111 & 35 & 60 & 21.00 & 8 & 16.78 & 19.78 & 1.64 & 0.56 & Aceptable \\
\hline 7 & 126 & 33 & 68 & 22.44 & 9 & 8.46 & 33.08 & 5.43 & 0.45 & Aceptable \\
\hline 8 & 135 & 50 & 73 & 36.50 & 6 & 48.68 & 10.67 & 0.31 & 4.47 & Muy buena \\
\hline 9 & 141 & 47 & 76 & 35.72 & 7 & 23.20 & 15.63 & 0.94 & 1.92 & Muy buena \\
\hline 10 & & 33 & 70 & 23.10 & 7 & 56.11 & 12.36 & 0.31 & 5.21 & Muy buena \\
\hline 11 & 120 & 60 & 64 & 38.40 & 7 & 44.45 & 13.02 & 0.41 & 4.05 & Muy buena \\
\hline 12 & 128 & 30 & 69 & 20.70 & 8 & 8.33 & 27.15 & 4.53 & 0.43 & Aceptable \\
\hline 13 & 115 & 50 & 62 & 31.00 & 6 & 45.30 & 14.22 & 0.44 & 4.13 & Muy buena \\
\hline
\end{tabular}

La Figura 1 muestra el análisis gráfico para determinar el grado de correlación de la RMSSD-Slope con las variables de volumen, intensidad, carga total de entrenamiento y escala de Borg, mostrando los valores de $\mathrm{R}^{2}$.

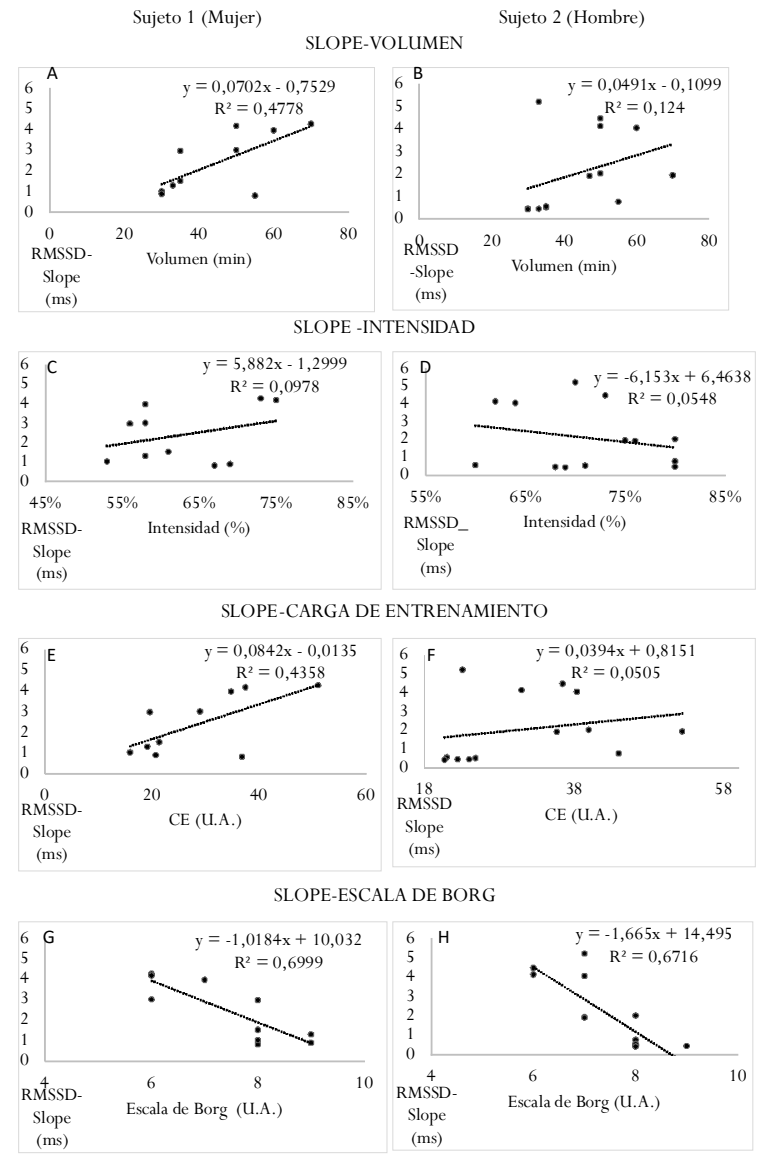

Figura 1. Relación lineal de los valores de la pendiente de recuperación de la raíz cuadrada de la media de la suma de los cuadrados de las diferencias sucesivas de los intervalos RR en ms (RMSSD-Slope) con volumen de las sesiones expresado en minutos (A y B); intensidad expresada en porcentaje (C y D); carga total de entrenamiento expresada en unidades arbitrarias (CTE) (E y F); Escala de Borg (G y H).
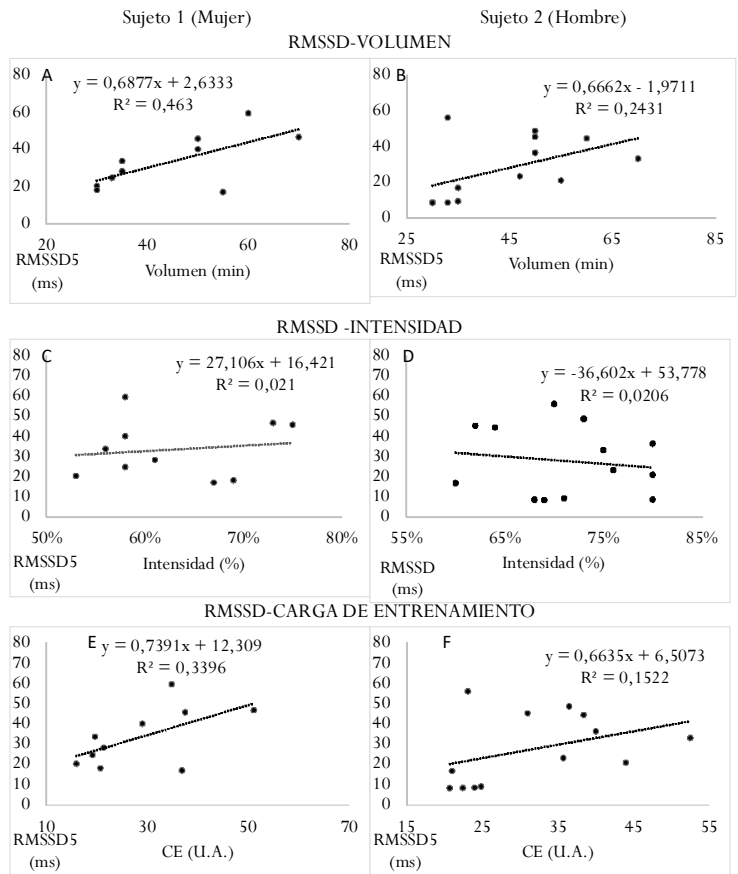

Figura 2. Relación lineal de los valores de la raíz cuadrada de la media de la suma de los cuadrados de las diferencias sucesivas de los intervalos RR en $\mathrm{ms}$, registrados durante 5 minutos (RMSSD 5) post-entrenamiento con volumen de las sesiones expresado en minutos (A y B); intensidad expresada en porcentajes (C y D); carga total de entrenamiento expresada en unidades arbitrarias (CTE) (E y F) 


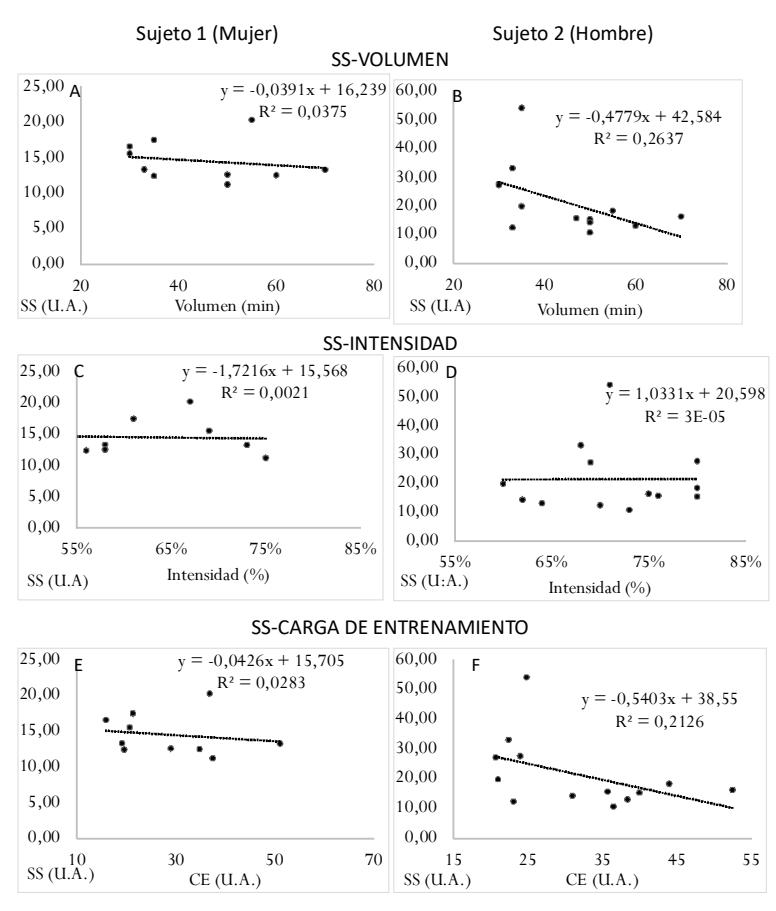

Figura 3. Relación lineal de los valores índice de estrés expresados en unidades arbitrarias (SS) post-entrenamiento con volumen expresado en minutos (A y B); intensidad expresada en porcentaje (C y D); carga total de entrenamiento expresada en unidades arbitrarias (CTE) (E y F)

Las Figuras 2 y 3 muestran el análisis gráfico para determinar el grado de correlación de la RMSSD5 y el $\mathrm{SS}$ respectivamente con las variables de volumen, intensidad, carga total de entrenamiento y escala de Borg, mostrando los valores de $\mathrm{R}^{2}$.

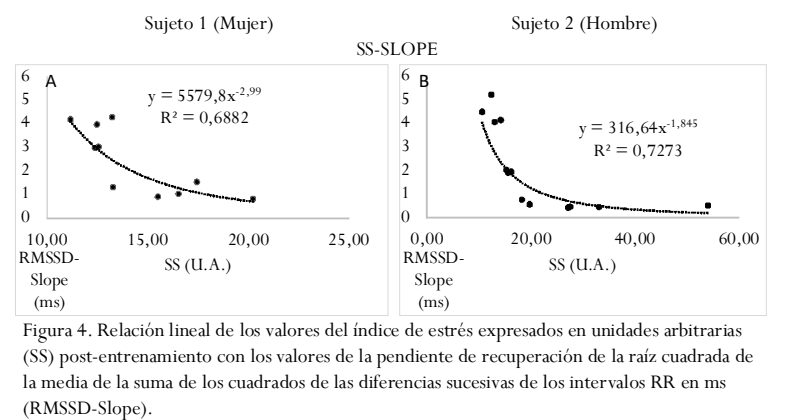
(RMSSD-Slope).

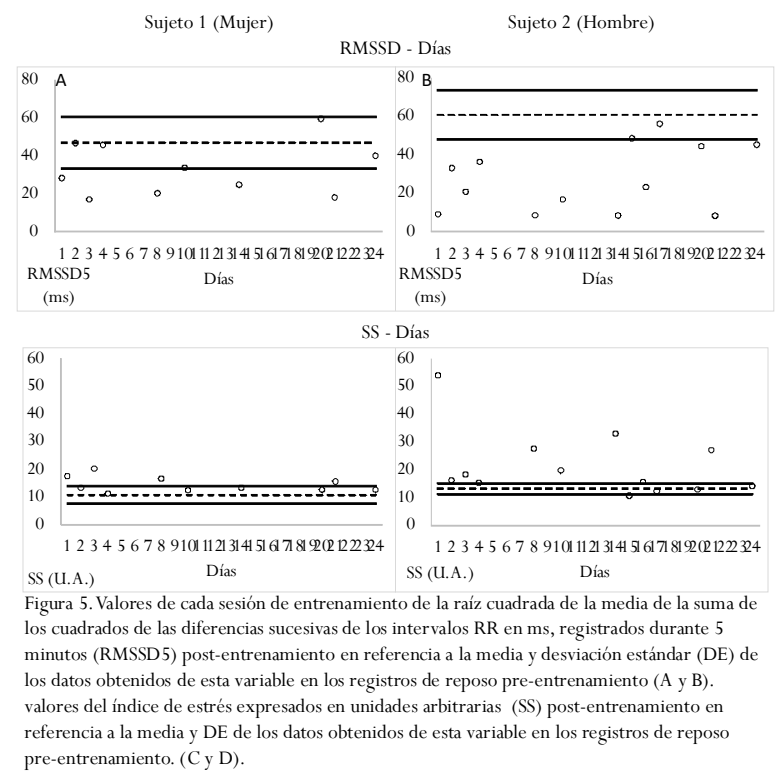

La Figura 4 refleja el análisis gráfico para determinar el grado de correlación entre RMSSD-Slope y el $\mathrm{SS}$, mostrando los valores de $\mathrm{R}^{2}$. Se observa que a mayor SS la RMSSD-Slope es más baja.

La Figura 5 muestra gráficamente los valores de la RMSSD-Slope y el SS post-entrenamiento en referencia a la media y la DE de los datos obtenidos para estas variables en los registros de reposo pre-entrenamiento.

\section{Discusión}

La principal contribución comprobar la operatividad práctica de la recuperación inmediata de la RMSSD como indicador de carga interna de entrenamiento en dos jugadores de bádminton.

Los valores de VFC medidos en estos dos deportistas (Tablas 1 y 2) se encuentran en los percentiles 10 (en el caso de la mujer) y 50 (en el caso del hombre) de las tablas de referencia de Medina et al. (2012). Los coeficientes de variación observados en estas tablas indican una alta variabilidad de las mediciones de cada día, lo que podría indicar las diferentes respuestas a las cargas de trabajo realizadas.

En la figura 1 se puede observar que no existe una relación lineal entre la RMSSD-Slope y las variables de entrenamiento (volumen, intensidad y carga total de entrenamiento), sin embargo observando la relación de la escala de Borg, medida aceptada de carga interna y la RMSSD-Slope, se puede comprobar que en ambos casos se da una correlación inversa (mujer $\mathrm{R}^{2}=-, 69$, hombre $\mathrm{R}^{2}=-, 67$ ), siendo a mayor valor de la percepción subjetiva del esfuerzo, menor pendiente de recuperación, con esto podemos concordar con Ruso-Álvarez et al. (2019) sugiriendo que la RMSSD-Slope es una herramienta válida como medida de carga interna.

Se observó que no hay una relación lineal de la RMSSD 5 y el SS con las variables de entrenamiento (volumen, intensidad y carga total de entrenamiento) (Figuras 2 y 3). Esta falta de relación iría en favor de que tanto la RMSSD-Slope como la RMSSD5 y el SS estén indicando carga interna, es decir, la respuesta individual a cada sesión de entrenamiento. Esta apreciación es importante porque en la literatura muchos artículos se centran en buscar correlaciones entre variables de carga externa y carga interna de entrenamiento (McLaren et al., 2018). Sin embargo, no sería razonable que esto suceda ya que precisamente el concepto de carga interna implica respuestas diferentes a las mismas cargas externas, por lo que no debiera haber una 
correlación entre ambas variables. Por ello, la RMSSDSlope parece ser un buen indicador de carga interna al presentar diferentes valores para las mismas intensidades de esfuerzo (Naranjo et al., 2019).

No hay una correlación entre los valores de SS y RMSSD con el volumen de los entrenamientos, lo cual sugiere que estas medidas son un indicador de CIE.

Si la RMSSD-Slope y el SS expresan de alguna manera la carga interna de entrenamiento, deberán hacerlo de forma inversa dado que el primero expresaría la respuesta del sistema parasimpático en la recuperación mientras que el segundo expresaría el impacto estresor producido por la carga de trabajo sobre el sistema simpático. De hecho, esto es lo que se observa en la figura 4.

En la Figura 5 observamos el comportamiento de la RMSSD5 y el SS en cada sesión de entrenamiento en relación con los valores basales, expresados como media (línea discontinua) y DE (líneas continuas). De esta forma se puede observar cómo las diferentes sesiones de entrenamiento producen respuestas diferentes, encontrándose algunas que prácticamente no alteran los valores basales y otras que suponen una alteración mucho mayor. También puede observarse, por ejemplo, cómo en general las sesiones de trabajo suponen un impacto estresor mayor en el hombre que en la mujer.

Nieto-Jimenez et al., (2020) analizaron la variabilidad de la frecuencia cardiaca en el control del entrenamiento en un corredor de Ironman, encontrando que los registros de las variables RMSSD y el SS proporcionan información para identificar cambios en las respuestas al entrenamiento, por ello es importante que los entrenadores tengan control sobre las respuestas a la carga de entrenamiento para evitar que el atleta tenga una mala adaptación al mismo (Andrade et al., 2020).

Solo encontramos tres trabajos en los que se estudian los cambios de la variabilidad de la frecuencia cardiaca en jugadores de bádminton. En uno de ellos se explica que a medida que el sujeto estudiado fue acumulando partidos en un periodo de tiempo corto se encontró un descenso progresivo de todos los valores del dominio de tiempo a medida que se disputan los partidos. En el caso del gráfico de Poincaré los autores describen que el diámetro transversal se reduce, mientras que el longitudinal se modifica menos, lo cual indica que existe una reducción de la actividad parasimpática y un aumento de la actividad simpática. Es por eso que llegaron a la conclusión de que en situaciones donde se acumula actividad, el sujeto presenta un comportamiento autónomo inclinado hacia un predominio simpático debido a la reducción de la actividad parasimpática (Garrido et al., 2009). Después de esto se realizó un estudio a 19 jugadores durante 2 o 3 días en el que se hizo un registro al inicio de la competencia y al finalizar cada uno de los encuentros, estos registros posteriores se realizaron durante los primeros 30 minutos de finalizados los partidos, se encontró en los valores del dominio de tiempo una reducción significativa de la VFC después de los partidos. En cuanto a los valores del gráfico de Poincaré, observaron un descenso en la ratio SD1/ SD2 a medida que los encuentros aumentaban. Con esto concluyeron que la acumulación de trabajo físico puede afectar la manera en la que el ejercicio induce cambios en el control simpático/parasimpático (Garrido et al., 2011). Por último, Ruso-Álvarez et al. (2019) al analizar la información de jugadores jóvenes de bádminton en tres partidos durante una competición de gran nivel, encontraron que mientras progresaba la cantidad de juegos, la media de las RMSSD-Slopes son más bajas. Esto sugiere que la RMSSD-Slope es un buen indicador de carga interna.

La principal limitación de este estudio fue, precisamente, que se trata de un estudio de caso y que, por tanto, los hallazgos expresados no pueden generalizarse. Sin embargo, aportan una línea de trabajo interesante para continuar con muestras suficientes de sujetos. Otra limitación podría ser el hecho de que las sesiones de entrenamiento no son las mismas en ambos sujetos, sin embargo, nos pareció interesante poder aportar la misma información en un deportista masculino y en otro femenino.

\section{Conclusión}

En conclusión, las mediciones de la VFC de cinco minutos después del entrenamiento fundamentan el uso de la RMSSD-Slope y el SS como indicadores de la carga interna de entrenamiento que reflejan la respuesta individual de los atletas al estímulo. La utilización de la RMSSD-Slope podría ser un método práctico, no invasivo y de manejo sencillo que puede ser utilizado por los preparadores físicos y entrenadores.

\section{Referencias}

Andrade,A. D., Sirnim, M., Kassiano,W., Palao, J. M., de Jesus, K., Maia, N.,Assumpção, C., \&Araripe Medeiros,A. I. (2020). Do differences between the training load perceived by elite beach volleyball players and that planned by coaches affect neuromuscular function? Retos, 83, 632-636. 
Banister, E.W., \& Calvert,T.W.(1980). Planning for future performance: implications for long term training. Canadian Journal of Applied Sport Sciences. Journal Canadien Des Sciences Appliquees Au Sport, 5(3), 170-176.

Bisschoff, C. A., Coetzee, B., \& Esco, M. R. (2018). Heart rate variability and recovery as predictors of elite, African, male badminton players' performance levels. International Journal of Performance Analysis in Sport, 18(1), 1-16. https://doi.org/ 10.1080/24748668.2018.1437868

Borg, G.A.V. (1954). Psychophysical bases of perceived exertion. Plastic and Reconstructive Surgery, 14(5), 377-381. https:/ / doi.org/10.1249/00005768-198205000-00012

Buchheit, M. (2014). Monitoring training status with HR measures: do all roads lead to Rome? Frontiers in Physiology, 5, 73. https:/ /doi.org/10.3389/fphys.2014.00073

Cabello Manrique, D., \& González-Badillo, J.J. (2003). Analysis of the characteristics of competitive badminton. British Journal of Sports Medicine, 37(1), 62-66. https://doi.org/10.1136/ bjsm.37.1.62

Foster, C. (1998). Monitoring training in athletes with reference to overtraining syndrome. In Med. Sci. Sports Exerc (Vol. 30, Issue 7). https://journals.lww.com/jnpt/00005768199807000-00023.fulltext

Garrido Esquivel,A., de la Cruz Torres, B., Garrido Salazar, M.A., Medina Corrales, M., \& Naranjo Orellana, J. (2009).Variabilidad de la frecuencia cardiaca en un deportista juvenil durante una competición de bádminton de máximo nivel. Revista Andaluza de Medicina del Deporte, 2(2), 70-74. https: / / www.redalyc.org/articulo.oa?id=323327658006

Garrido Esquivel,A., De la CruzTorres, B., Medina-Corrales, M., Garrido Salazar, M. A., \& Naranjo Orellana, J. (2011). Heart rate variability after three badminton matches. Are there gender differences? Archivos de Medicina Del Deporte, 28(144), 257 264.

Goldberger, J. J., Le, F. K., Lahiri, M., Kannankeril, P. J., Ng, J., \& Kadish, A. H. (2006). Assessment of parasympathetic reactivation after exercise. American Journal of Physiology - Heart and Circulatory Physiology, 290(6). https://doi.org/10.1152/ ajpheart.01118.2005

Hayes, P. R., \& Quinn, M. D. (2009). A mathematical model for quantifying training. European Journal of Applied Physiology, 106(6), 839-847. https://doi.org/10.1007/s00421-009-1084-8

Javorka, M., •ila, I., Balhárek, T., \& Javorka, K. (2002). Heart rate recovery after exercise: Relations to heart rate variability and coplexity. In Brazilian Journal of Medical and Biological Research (Vol. 35, Issue 8, pp. 991-1000). Associacao Brasileira de Divulgacao Cientifica. https://doi.org/10.1590/S0100879X2002000800018

Malik, M., Camm, A. J., Bigger, J.T., Breithardt, G., Cerutti, S., Cohen, R. J., Coumel, P., Fallen, E. L., Kennedy, H. L., Kleiger, R. E., Lombardi, F., Malliani, A., Moss, A. J., Rottman, J. N., Schmidt, G., Schwartz, P.J., \& Singer, D.H. (1996). Heartrate variability. Standards of measurement, physiological interpretation, and clinical use. In European Heart Journal. https: / /doi.org/10.1093/oxfordjournals.eurheartj.a014868

McLaren, S. J., Macpherson, T.W., Coutts, A. J., Hurst, C., Spears, I. R., \&Weston, M. (2018).The Relationships Between Internal and External Measures of Training Load and Intensity in Team Sports: A Meta-Analysis. In Sports Medicine (Vol. 48, Issue 3, pp. 641-658). Springer International Publishing. https:// doi.org/10.1007/s40279-017-0830-z

Medina Corrales, M., De la CruzTorres, B., Garrido Esquivel,A., Garrido Salazar, M.A., \& Naranjo Orellana, J. (2012). Normal values of heart rate variability at rest in a young, healthy and active Mexican population. Health, 04(07), 377-385. https: / /doi.org/10.4236/health.2012.47060

Melanson, E. L., \& Freedson, P. S. (2001).The effect of endurance training on resting heart rate variability in sedentary adult males. European Journal of Applied Physiology, 85(5), 442-449. https: / /doi.org/10.1007/s004210100479

Naranjo Orellana, J., De La CruzTorres, B., Cachadiña, E. S., De Hoyo, M., \& Domínguez Cobo, S. (2015). Two new indexes for the assessment of autonomic balance in elite soccer players. International Journal of Sports Physiology and Performance, 10(4), 452-457. https://doi.org/10.1123/ijspp.2014-0235

Naranjo Orellana, J., Nieto-Jiménez, C., \& Ruso-Álvarez, J. F. (2019). Recovery Slope of Heart RateVariability as an Indicator of InternalTraining Load. Health, 11(02), 211-221. https: / / doi.org/10.4236/health.2019.112019

Nieto-Jimenez, C., Ruso-Álvarez,J., Pardos-Mainer, E., \& Orellana, J. N. (2020). Heart RateVaribility in the training monitoring of an Ironman runner. A case study. Retos, 37, 339-343.

Reina, M., Mancha-Triguero, D., García-Santos,D., García-Rubio, J., \& Ibáñez, S. J. (2019). Comparación de tres métodos de cuantificación de la carga de entrenamiento en baloncesto. [Comparison of three methods of quantifying the training load in basketball]. RICYDE. Revista Internacional de Ciencias Del Deporte, 15(58), 368-382. https://doi.org/10.5232/ ricyde2019.05805

Reina, M., Rubio, J. G., Antúnez, A., \& Ibáñez, S. J. (2020). Comparison of internal and external load in official 3 vs. 3 and 5 vs. 5 female basketball competitions. Retos, 37, 400-405.

Ruso-Álvarez, J. F., Nieto-Jiménez, C., Muñoz-López, A., \& Orellana, J.N. (2019). Utility of the «RMSSD-Slope» to Assess the Internal Load in Different Sports Situations. Health, 11(06), 683-691. https://doi.org/10.4236/health.2019.116057 Tulppo, M., Hautala,A., Makikallio, T., Laukkanen, R., Nissila, S., Hughson, R., \& Huikuri, H. (2003). Effects of aerobic training on heart rate dynamics in sedentary subjects. Journal of Applied Physiology (Bethesda, Md./ : 1985), 95, 364-372. https:// doi.org/10.1152/japplphysiol.00751.2002

Tulppo, M., Makikallio,T.H.,Takala,T.E., Seppanen,T., \& Huikuri, H.V. (1996). Quantitative beat-to-beat analysis of heart rate dynamics during exercise. The American Journal of Physiology, 271(1 Pt 2), H244-52.https://doi.org/10.1152/ ajpheart.1996.271.1.H244 